\title{
LA MUJER EN LA UNIVERSIDAD ESPAÑOLA
}

\author{
Ángeles Laorden y Pilar Giménez
}

El artículo analiza la situación de la mujer en la universidad española actual y la historia de su participación en ella. Se incluyen datos sobre la evolución de la participación de las mujeres en las distintas profesiones. Finalmente, se señala la situación actual del movimiento feminista en las universidades catalanas. 


\section{El por qué de un artículo sobre mujer y Universidad}

En cualquier análisis que se realiza sobre la situación de la mujer en nuestra sociedad, se alude siempre al papel que tradicionalmente ha jugado la educación (y que de hecho hoy sigue jugando) como medio para mantener sometida a la mujer y justificar al mismo tiempo este sometimiento, contribuyendo a la configuración de roles sociales diferentes en función del sexo.

Sin embargo, el estudio que se ha realizado de todas las etapas educativas respecto a la situación de discriminación que en ellas sufre la mujer, es mucho menos completa en lo que se refiere a Universidad. Evidentemente, la incorporación tardía de la mujer a los estudios superiores, así como la crisis actual de la función de la Universidad, son factores que explican en parte esta falta de análisis, pero que no pueden obviar la necesidad que hoy tenemos todas las mujeres de plantear la lucha en contra de la discriminación, en todos y cada uno de los sectores sociales, en todas y cada una de las facetas de nuestra vida diaria, y por tanto, saber también que no es posible una liberación de la mujer sin una transformación total de las estructuras sociales, transformación en la que la Universidad es una pieza clave.

En esta transformación de la institución universitaria, habrá un sentido favorable o desfavorable a nuestra lucha en la medida en que la mujer intervenga en el proceso, planteando no sólo sus reivindicaciones específcas, sino también aportando todo el potencial que como personas hasta hoy se nos ha negado.

\section{El papel de la Universidad}

No es nuestro objetivo en este artículo el realizar un estudio detallado de cuáles son las funciones que la Universidad realiza dentro de la actual sociedad, pero sí analizar el papel que juegan en cuanto a la mujer. 
En el actual momento histórico la función que a la Universidad le es asignada por las clases dominantes es, por una parte, reproducir la ideología de estas clases para seguir manteniendo el mismo tipo de estructura social, y por otra, la formación de cuadros técnicos necesarios en el sistema productivo.

Tanto un aspecto como el otro, implican la división de clases, pero también la división de sexos, como lógica inherente al sistema. Factores que más tarde analizaremos, como la tardía y menor incorporación de la mujer a la Universidad, su orientación hacia «carreras femeninas», su poca actividad profesional una vez acabada la carrera, no son más que consecuencias de la necesidad que el sistema tiene de reproducir una división de sexos que le permita seguir existiendo.

La educación y el acceso a ella de la mayoría de la población, como exigencia todavía no conseguida, es un aspecto que se hace más patente en lo que se refiere a la mujer, y que se acentúa a medida que vamos superando las diferentes etapas educativas hasta llegar a la Universidad, pues aquélla sufre una selectividad no sólo clasista, sino también una selectividad en función del sexo.

La crisis institucional de la Universidad por un lado, y el auge del movimiento feminista por otro, han hecho surgir a la luz la discriminación que la mujer sufre en la última etapa del sistema educativo. En primer lugar nosotras llegamos a la Universidad en inferioridad de condiciones respecto a los hombres, porque el punto de partida ya ha sido diferente. Cuantitativamente el número de mujeres pasa de ser igual e incluso superior al de los hombres en los primeros niveles de la enseñanza, para ir disminuyendo progresivamente hasta llegar a la Universidad. Cualitativamente nuestra «actitud» o «disposición» es menos activa que la masculina porque los estímulos de estudio y de cualificación profesional han sido distintos.

Los mecanismos que dan lugar a esta situación de inferioridad, y teniendo en cuenta que legalmente no existe ningún impedimento para el acceso de la mujer a la Universidad, escapan al ámbito puramente universitario. Comienzan en la familia, continúan en la escuela y, en definitiva, se extienden a todos los ámbitos sociales que van configurando unos roles diferentes según el sexo identificando valores como actividad, capacidad de raciocinio... con «lo masculino» y la pasividad, intuición, emotividad... (léase histeria en el lenguaje masculino) será identificable con «lo femenino». Estos valores han nacido de las funciones diferentes $-\mathrm{y}$ en ocasiones antagónicas - que la sociedad de clases configura para cada sexo, pero al mismo tiempo son estos valores los que «justifican» la posición de dominio de un sexo sobre el otro. La esfera pública será el mundo 
masculino, y por lo tanto más prestigiado en todos los aspectos; la esfera privada —el hogar- corresponderá al mundo de las mujeres.

La dominación del sexo fernenino por el masculino ha estado históricamente ligada a la división de clases. En todas las sociedades basadas en la propiedad privada de los medios de producción, la mujer, en cuanto mujer, ha tenido un rol subalterno, rol que se fundamenta en la exclusión de las féminas del proceso productivo extradoméstico, y se apoya en la coacción ideológica mediante la cual las mujeres debemos aceptar pasivamente nuestro destino. Es aquí donde la Universidad juega un papel que a primera vista puede parecer contradictorio, porque por un lado «prepara» a la mujer profesional y culturalmente, pero al mismo tiempo esta educación se sigue impartiendo con los mismos criterios que «justifican» la inferioridad femenina y por lo tanto adecuan la superestructura ideológica a unas nuevas necesidades económicas. La inserción del «sexo débil» en el proceso productivo es un imperativo de los tiempos, y por lo tanto hay que educar a la mujer para «hacer» algo, aun cuando para «ser» algo necesite cumplir el papel de esposa y madre que continúe garantizando la conservación de la especie (mitificación de la maternidad) y el mantenimiento de la misma, supliendo con su trabajo doméstico una serie de servicios que el Estado no asegura. Es decir, que la Universidad reproduce una ideología sexista y funciona también de una manera sexista. Que funciona de una manera sexista lo demuestra la discriminación entre las propias profesionales de la Universidad, pues aunque la mujer supone el $68 \%$ del total de los enseñantes (en la preescolar - categoría menos remunerada y menos prestigiada - la presencia de la mujer enseñante es del $98 \%$ ), en la Universidad tan sólo el $9 \%$ de enseñantes es del sexo femenino, y de éstas, el porcentaje es mayor entre los contratados que entre adjuntos y catedráticos. En España no existe ni una sola mujer decano o rector.

Así, pues, la selectividad sobre el sexo femenino está íntimamente ligada al papel que la mujer tiene dentro de la familia como reproductora de la fuerza de trabajo, luego tanto económica como políticamente su incorporación al proceso productivo no ha sido hasta ahora vital, y mucho menos que en el caso que esta incorporación se produzca sea en condiciones de igualdad con el hombre, en cuanto a formación y posibilidades se refiere. En definitiva, si actualmente hay una tendencia a la desvalorización del título universitario como valor de cambio, en la mujer su título ha tenido siempre un valor mucho menor en el mercado de trabajo, excepto en épocas excepcionales (guerras, etcétera) en que la coyuntura lo exigía, y siempre como mano de obra de reserva. 


\section{Historia de la discriminación}

No es de extrañar que en España hasta 1910 las mujeres no pudieran matricularse en la Universidad sin el consentimiento previo de las autoridades, y que en el curso de 1919-1920 sólo hubiera 439 mujeres estudiando en la Universidad, lo que representaba el $2 \%$ de la población universitaria.

Una de las razones del escaso número de universitarias fue sin duda la falta de escuelas secundarias que las preparasen para los estudios superiores, pero el obstáculo más importante era la gran hostilidad que despertaba la simple idea de una educación superior para la mujer. Valga como muestra de esta hostilidad la crónica de España Moderna ${ }^{1}$ en la que se plantea que aunque el éxito de las mujeres en el hogar y en la escuela es innegable, en la Universidad son inferiores a los hombres.

Ortega, uno de los filósofos más misóginos, nos define como seres confusos, con una forma de humanidad inferior a la varonil. Es bastante ejemplificador lo que Madariaga escribe el año 1934 sobre la mujer: ${ }^{2}$ la idea de la mujer culta para mayor goce del marido, para entretenerle y solazarle con sus conocimientos, pero sin que éstos pongan en peligro la superioridad de aquél, su autoridad. La mujer tendrá reservadas unas especiales «facultades» que le permitan desarrollar sus condiciones innatas, en definitiva la eterna idea de mantener la división de roles de los sexos; es curioso que al finalizar el punto dedicado a la educación de la mujer, Madariaga llega a decir: «de ello depende el porvenir de la República»...

El proceso de industrialización comenzado en el resto de países europeos en las postrimerías del siglo XIX no tiene la misma evolución en España; serán sólo Cataluña y el País Vasco donde tendrá lugár un cierto desarrollo industrial. Este retraso económico incide lógicamente en el retraso cultural, y si a finales del siglo xIx existían en España un $71 \%$ de mujeres analfabetas, en 1930 sólo el 3,8 \% de las mujeres pasan de la escuela primaria. El escaso tiempo de vida de la II República permitió incorporar a la mujer en una mayor proporción en el campo educativo,

1. «La mujer es esencialmente un ser receptivo, pero cuando se trata de facultades creadoras tiene que contentarse con el segundo rango.» F. Araujo, «La coeducación de los sexos», España Moderna 15, núm. 176 (agosto 1903).

2. «Hay una minoría de mujeres que, ya por tendencia viriloide, ya por azar, no se casan y prefieren ocupaciones de tipo masculino... Las mujeres representan para la sociedad un valor civilizador inestimable, puesto que a ellas se debe la tendencia al refinamiento, al bienestar, a los modales urbanos y a las artes de la vida.»

«Tiene por objeto estimular, desarrollat y afinar las tendencias innatas femeninas a la gracia, al refinamiento y a las artes de la vida.» Salvador de Madariaga, Anarquía o jerarquia (Madrid: Editorial Aguilar, 1970), 3a. ed., p. 287. 
implantando la coeducación y abriendo el acceso a la mujer a las profesiones liberales.

El derrocamiento de la II República supuso un retroceso para la mujer; se produce un decrecimiento en la tasa de actividad femenina -pasa de un $9 \%$ en 1939 a un 8,3\% en 1940_, acentuándose el papel de la mujer como agente reproductor, en parte para compensar las bajas producidas por la guerra civil, y en parte por la falta de puestos de trabajo. Esta tendencia es fomentada por la nueva legislación —el Fuero del Trabajo de 1938 prometía «liberar a la mujer de la esclavitud del taller y de la fábrica»- e ideología oficial, en la que la escuela jugará un papel fundamental; se produce un retroceso en la enseñanza, con prioridad de los centros privados sobre los estatales y el sistema educativo se convierte en minoritario y elitista; en estos años la tasa de educación femenina es muy baja, situación que va cambiando paulatinamente cuando a partir de 1949 se inicia el Plan de estabilización, que cierra la etapa autárquica y abre un tímido camino para la industrialización. En 1962 la tasa de población activa femenina era de un $13,5 \%$, porcentaje que va aumentando sobre todo al incrementarse puestos de trabajo en el sector terciario (actualmente la población activa femenina supone en España un $25 \%$ ); las necesidades de adaptar el sistema educativo al proceso de desarrollo del capital monopolista de Estado, dan lugar a la Ley General de Educación con la implantación de la enseñanza obligatoria y gratuita —en teoría - hasta los 14 años (EGB). Aunque aparentemente esto repercute positivamente en la mujer, puesto que aumenta su presencia en la enseñanza media y se eleva en general su nivel cultural, la doble titulación establecida al final de la EGB desvía a la gran masa de mujeres a la Formación Profesional, y dentro de ésta a las ramas más marginales y decorativas, lo que proporciona una mano de obra en el mercado laboral barata, secundaria y transitoria.

\section{Situación actual}

Las necesidades productivas, así como la presión de la sociedad en favor de una educación más igualitaria, han configurado nuevos valores para la función de la mujer. Ya no será vergonzoso el que ésta tenga unos estudios superiores, si bien nunca podrán constituir un obstáculo en su papel de esposa y madre. No existen teóricamente discriminaciones en el acceso a los estudios, pero el número de mujeres matriculadas en relación a los hombres es menos en los estudios superiores, y aunque como vercmos 
en el siguiente apartado, esta tendencia tiende a disminuir en los últimos años, el camino hacia la simple igualdad numérica será largo.

El otro aspecto que no se puede dejar de analizar es la función que los estudios universitarios tienen para la mujer. Si para el hombre una carrera superior supone la posibilidad de introducirse en el proceso productivo con un status social y económico elevado, para muchas mujeres, teniendo en cuenta que tan sólo alrededor del $50 \%$ acaban ejerciendo profesionalmente, su paso por la Universidad implica una especie de compás de espera que las encaminará hacia la meta del hogar con una mayor «cultura», pero sin que haya variado un ápice su explotación.

El número de mujeres que inician una carrera es mucho mayor que el de las que acaban. Entre estas últimas es numeroso el grupo de las que renuncian a su profesión en función de la dedicación exclusiva a su papel de esposa y madre, y así no es de extrañar que en 1974 - según los datos del censo de la población activa- solamente el $56 \%$ de las mujeres que poseían estudios superiores eran activas, y de los técnicos superiores sólo el 9,6\% eran mujeres.

Las mujeres que intentan llevar una vida profesional activa tendrán que competir por la consecución de un puesto de trabajo, viéndose en desventaja frente a su compañero de estudios de igual calificación. En primer lugar han recibido una formación que se adapta muy mal a las necesidades de la economía, porque mientras el hombre recibe una formación cada vez más técnica, las profesiones llamadas «femeninas» conducen a los sectores de actividad más desvalorizados y a los puestos de trabajo menos calificados y peor retribuidos. La división del trabajo da lugar a un doble metcado laboral que sitúa en inferioridad a la mujer a la hora de su inserción profesional, por las posibilidades limitadas de acceso a los empleos generalmente ocupados por los hombres, e incluso cuando no se estipula el sexo, se produce de hecho una doble oferta paralela, una para hombres y otra para mujeres, en la que el mismo título es considerado según el sexo del que lo posee. Cuando la mujer está en posesión de un empleo, de nuevo se la vuelve a discriminar; su salario es siempre inferior al del hombre, y con igual cualificación su sueldo es relativamente inás elevado en las ramas de actividad mixta que en las que tienen un elevado porcentaje de mano de obra femenina, y así, en las industrias donde las mujeres son mayoritarias, los salarios se desvalorizan, lo que hace que los hombres las abandonen.

Todos estos factores hacen aumentar las contradicciones de la supuesta igualdad de sexos en la Universidad y la práctica social de la mujer universitaria que se ha visto discriminada antes, en y después de la Universidad. $\mathrm{Ha}$ accedido al universo cultural de los hombres, pero tendrá 
que estar siempre dentro del papel secundario para el que ya nació marcada por el hecho de ser mujer.

Evolución de la matricula en las distintas facultades:

las carreras temeninas

En nuestro país, el nivel de formación se ha elevado como consecuencia de las necesidades del desarrollo - con la complejidad creciente de la producción técnica, pero también porque las exigencias de la población de acceder a la «cultura» son cada día mayores. En estas necesidades, tanto económicas como psicológicas, la mujer -aunque en menor medidase ha incorporado progresivamente al sistema educativo. La Universidad es la última etapa, y por lo tanto la más costosa de conquistar por el sexo femenino. Casos como el de Concepción Arenal, la gran jurista española que tuvo que disfrazarse de hombre para acceder a las aulas, pertenecen ya al campo de la historia de la discriminación; no en vano ha transcurrido un siglo.

Sin embargo, en el curso 1973-1974, la mujer constituía el $32 \%$ del alumnado total de educación universitaria, incluyendo escuelas de profesorado de EGB, Facultades y Colegios Universitarios, Escuelas de Estudios Empresariales, Escuela de Arquitectura e Ingeniería y Escuelas T'écnicas Superiores. Este dato demuestra que ha habido una disminución de la presencia femenina en el paso de la Enseñanza Media -donde supone aproximadamente la mitad - a la Universidad; la diferencia ha sido desviada o bien al segundo ciclo de Formación Profesional o bien al mercado de trabajo directamente.

Según los datos recogidos en el anexo (obtenidos de las estadísticas publicadas por el Ministerio de Educación y Ciencia) para el curso 19731974, la igualdad numérica entre ambos sexos es todavía una utopía. El número de alumnos matriculados en las diversas facultades ha crecido enormemente en los últimos años y de modo proporcional, pero ligeramente superior, lo ha hecho el número de alumnas. Las carreras preferidas a nivel general han cambiado: Derecho, la carrera con más alumnos en los años cuarenta, ocupa hoy un discreto cuarto lugar. La moda o preferencia no tiene una relación directamente proporcional al status o prestigio profesional que dicha carrera proporciona, si bien éste es el factor dominante. Sin embargo, puede establecerse: que el número de alumnos matriculados y el porcentaje mínimo de mujeres que registran algunas facultades, tiene una relación directa con las necesidades del sistema, con el valor de cambio del propio título. Es sintomático al respecto el caso de la Facultad de 
Medicina: en países que, como la URSS, dicho título no proporciona unas ventajas especiales de tipo económico o social, el porcentaje de mujeres es elevadísimo, mientras que en los países donde esta profesión goza de gran prestigio -España o USA-, el número de alumnos es alto y el porcentaje de mujeres mínimo.

Existen carreras, las denominadas femeninas, en las que la mayoría de alumnos son mujeres; éstas, ya tradicionalmente, son: Farmacia, Filosofía y Magisterio (hoy escuela de EGB). La primera posibilita la entrada en un laboratorio, trabajo que al ser de «detalle» se considera óptimo para la mujer, o bien, si tiene dinero, puede montar una farmacia, con un horario que por su flexibilidad puede compatibilizarse con las tareas del hogar. En ningún caso se trataría de carreras o trabajos de responsabilidad. El otro campo clásico sería el de la Enseñanza, tradicionalmente mal remunerado y desprovisto de prestigio; ya se sabe que las mujeres saben tratar a los niños... En general, hoy las mujeres tienen abiertas las puertas de los estudios humanísticos, a los que desgraciadamente se les niega toda importancia, mientras que las carreras técnicas, hoy las más prestigiadas, están totalmente vedadas a las mujeres; es el reflejo de la barrera que se impone a la mujer a cualquier forma de conocimiento científico, racional y práctico, forma de conocimiento más valorada en la sociedad tecno crática.

\section{La lucha feminista en la Universidad}

Las «Jornades Catalanes de la Dona», que se celebraron en Barcelona en mayo de 1976, puede decirse que tuvieron una importancia decisiva para el movimiento de mujeres en Cataluña. Supusieron la culminación de un proceso de reivindicaciones feministas, un gran paso hacia la palestra pública y la apertura de una nueva etapa en el movimiento feminista en general.

$\mathrm{Si}$, como decimos, la importancia de estas jornadas en el movimiento feminista y su repercusión a nivel ciudadano fue innegable, ¿qué repercusión tuvieron a nivel estrictamente universitario? Las «Jornades» se celebraron en el Paraninfo de la Universidad Central y a ellas acudieron gran número de estudiantes y profesoras; sin embargo, el Paraninfo fue una mera localización geográfica y las universitarias que acudieron no lo hicieron como tales, sino como mujeres interesadas, en mayor o menor grado, en sus problemas.

En las luchas llevadas a cabo por la Universidad en los últimos años, la mujer ha participado siempre de una manera activa, pero, dentro de 
estas luchas, en el contexto general de transformación de la institución universitaria, son prácticamente inexistentes tanto reivindicaciones femeninas como planteamientos feministas. Ello es debido fundamentalmente a dos factores:

Por un lado, la conciencia ampliamente extendida de que en la Universidad, propiamente, no existe una discriminación contra la mujer, que el terreno universitario es una especie de gbetto privilegiado donde la mujer es igual al hombre. Evidentemente, esta postura adolece de una falsa concepción de lo que es la Universidad en sí, al aislarla del contexto global en el que está inserta, y sobre todo al considerar la situación de inferioridad de la mujer como una discriminación que se produce por parcelas y no de una manera total, sobre la personalidad femenina. Ya hemos visto cómo la mujer sufre una selectividad previa y una posterior a la Universidad, y esto es precisamente lo que produce una contradicción constante entre su práctica social y su situación como universitaria.

El segundo factor, que podría explicar la falta de conciencia feminista dentro de la Universidad, sería la actitud de los partidos de izquierda (hoy mayoritarios en la Universidad) respecto a la problemática de la mujer. Su atención ha sido prácticamente nula y aunque hoy en día la situación está cambiando a nuestro favor, hasta hoy ha tenido un peso negativo.

De todo lo explicado anteriormente se deduce que las mujeres en la Universidad, además de seguir participando como hasta ahora en la lucha global, han de dar a esta participación un carácter feminista, tanto en reivindicaciones inmediatas, denuncia de casos concretos de discriminación, etcétera, como en la inclusión de planteamientos feministas en toda alternativa de transformación de la Universidad.

Por ello pensamos que toda organización de mujeres en la Universidad (organización hoy necesaria) debe plantearse dos frentes de trabajo: uno, el movimiento feminista en general, coordinándose por lo tanto con el resto de grupos feministas y participando en las campañas generales que se planteen, y el otro, el estrictamente universitario, donde la función social de la mujer en cada carrera ha de ser un eje principal. Por poner algunos ejemplos: las mujeres de Medicina tienen un amplio campo de acción en todo lo referente a planificación familiar, contracepción, aborto...; en Derecho la situación de discriminación legislativa, en diferentes campos; en EGB el planteamiento de una educación no sexista... En definitiva, se trata no sólo de que la transformación de la Universidad implique como condición sine qua non la desaparición de toda discriminación por razón de sexo, sino de que desde la propia Universidad se contribuya de una manera activa a la construcción de una sociedad nueva, donde la mujer deje de ser un ciudadano de segunda categoría. 


\section{La actual situación organizativa}

En el momento de redactar estas páginas (noviembre de 1977) la situación a nivel organizativo es la siguiente:

Existe un grupo llamado AUPEM: Asociación Universitaria Para el Estudio de los Problemas de la Mujer. Esta asociación se propone como fines el «análisis y denuncia de la discriminación que sufre la mujer en todas las facetas: educación, familia, trabajo, sexualidad...».

En el terreno educativo AUPEM plantea: que se cumpla el principio de igualdad de acceso a la educación reconocido por la ley; fomento de la coeducación en todos los niveles y grados; profesorado mixto en la enseñanza privada; transformación del contenido de las asignaturas de formación doméstica y extensión a ambos sexos; eliminación de toda la propaganda que presenta a la mujer como mero objeto sexual y decorativo...

En cuanto a reivindicaciones concretas: a) guarderías suficientes y adecuadas, $b$ ) planning familiar y derogación de la norma penal que prohíbe la contracepción, etcétera.

Actualmente es el único grupo no coordinado dentro de los Grupos de Mujeres de Universidad, que en estos momentos es el mecanismo donde se agrupan los grupos que funcionan ya en las distintas facultades y que se coordinan directamente en la coordinadora de Barcelona.

En las asambleas de distrito realizadas se han planteado los puntos mínimos de carácter organizativo del futuro movimiento unitario de mujeres. Estos puntos programáticos serían:

1. Unitario: Esto es, que incluyera a todas las mujeres y grupos de cualquier tendencia que, en el campo universitario, estuvieran interesadas por los problemas de la mujer, fuera cual fuera su esfera de trabajo: estudiantes, profesoras, personal no docente.

2. Autónomo: No como un órgano de un futuro sindicato estudiantil, o de profesores, sino como un ente independiente cuyos miembros querrán formar parte o no de ellos, independientemente de su relación con la organización de mujeres. Autónomo de los partidos políticos, es decir, que las mujeres participarán como tales y votarán individualmente al margen de su adscripción a un partido.

3. Democrático: Por su propia esencia y carácter, por la misma condición de unitario y autónomo y por la admisión de todo tipo de tendencias en su seno.

4. Femenino: Éste quizás ha sido el punto más controvertido en las asambleas, pero al que ha acabado sumándose la práctica totalidad de mujeres. Se ha decidido excluir del organismo, de los grupos, a los univer- 
sitarios, por no estar afectados de manera directa en el problema, lo que no prejuzga su desinterés por el tema, sino que por el contrario se espera y se recabará ayuda a los compañeros en todos los casos en que se crea conveniente, sin que por ello pertenezcan al grupo.

5. Representativo $y$ de masas: Aspira a integrar a todas las mujeres que se hallen en la Universidad y a integrar todas las corrientes que se presentan en el campo de los movimientos feministas.

Todo ello ha sido fruto de un proceso de discusión, empezado el primer trimestre de curso, y hoy casi finalizado, en lo que hace referencia a la organización y que ha cristalizado en la creación de grupos de mujeres en muchas facultades del distrito y que se coordinan a nivel de Barcelona con la Coordinadora, y entre las distintas facultades por medio de Asambleas de distrito, estando aún en estudio el problema de la coordinación por representantes a nivel de universidad.

\section{Conclusión}

Es evidente que este artículo sólo esboza algunos aspectos de la situación de la mujer en la Universidad. La discriminación nos parece patente, pero el problema se sitúa no sólo constatando la discriminación y las causas de ésta, sino fundamentalmente ver en qué terreno debe situarse la lucha de las mujeres dentro de la Universidad, dentro por lo tanto del «reino» de los modelos masculinos del saber.

Creemos que desarrollar todo el potencial creativo de la mujer implica un más alto grado de formación y también otro tipo de formación. Pero, ¿cómo resolver la contradicción que se plantea al exigir una igualdad de conocimientos con el hombre, cuando los estímulos son diferentes y cuando además no estamos de acuerdo con la concepción y utilización que se hace de estos conocimientos?

La cuestión no afecta solamente a las mujeres universitarias. Es un debate abierto en todos los movimientos feministas, no tanto respecto a su razón de ser y existir, sino en cuanto a la eficacia de estos movimientos en transformar una sociedad machista y masculinizada.

En definitiva, ¿debemos lai mujeres cambiar esta sociedad introduciéndonos en ella, es decir, con las reglas de juego masculinas, o al rechazar estas reglas, nuestras opciones feministas hemos de imponerlas sin «ensuciarnos las manos» en unos terrenos en los que la competitividad, el poder de mando y la agresividad son las normas de conducta? ¿Han de estar los movimientos feministas presentes en los organismos de poder potenciando 
«Papers»: Revista de Sociología

el cambio también desde ellos, o nos hemos de limitar a presionar desde la base de la sociedad?

Construiremos un mundo diferente a partir de una lucha aislada, o bien las feministas hemos de estar en todos los terrenos de la sociedad, y por lo tanto también - ¿ por qué no? - interviniendo en el «mundo masculino» desde sus centros de decisión.

Los interrogantes en todo caso han de seguir abiertos. Colectivamente las mujeres no hemos encontrado aún nuestras señas de identidad. El camino para ello es el que ya hemos emprendido: aprender a partir de de nuestra propia lucha. 
APENDICE

Estadísticas correspondientes a las universidades catalanas en el curso 1973/1974

\begin{tabular}{lcccccc}
\hline \multicolumn{2}{c}{ Alumnos matriculados } & & \multicolumn{3}{c}{ Licenciados } \\
\cline { 5 - 7 } Total & Mujeres & $\%$ & & Total & Mujeres & $\%$ \\
\hline & & & & Total & & \\
31.941 & 12.213 & 38,18 & Central & 2.636 & 933 & 35,39 \\
10.347 & 3.807 & 36,79 & Autónoma & 933 & 121 & 41,01
\end{tabular}

Facultad de Ciencias

$\begin{array}{rrrlrrr}5.044 & 1.835 & 36,37 & \text { Central } & 384 & 177 & 46,09 \\ 2.147 & 789 & 36,74 & \text { Autónoma } & 47 & 13 & 27,75 \\ 50.746 & 18.547 & 36,54 & \text { España } & 2.866 & 940 & 32,79\end{array}$

Facultad de Económicas

$\begin{array}{rrrlrrr}4.490 & 622 & 13,85 & \text { Central } & 578 & 33 & 5,70 \\ 1.057 & 142 & 13,43 & \text { Autónoma } & 15 & 8 & 53,33 \\ 22.114 & 3.589 & 16,22 & \text { España } & 2.142 & 357 & 16,66\end{array}$

Facultad de Derecho

$\begin{array}{rrrlccc}4.960 & 1.301 & 26,22 & \text { Central } & 325 & 85 & 26,15 \\ 729 & 149 & 20,43 & \text { Autónoma } & - & - & - \\ 36.157 & 10.580 & 29,26 & \text { España } & 1.704 & 381 & 22,35\end{array}$

Facultad de Farmacia

$\begin{array}{ccclccc}3.257 & 1.725 & 59,26 & \text { Central } & 153 & 81 & 52,94 \\ - & - & - & \text { Autónoma } & - & - & - \\ 13.796 & 7.867 & 57,13 & \text { España } & 680 & 435 & 63,97\end{array}$

Facultad de Medicina

$\begin{array}{rrrlrrr}4.808 & 1.558 & 32,40 & \text { Central } & 609 & 213 & 34,97 \\ 2.720 & 744 & 27,35 & \text { Autónoma } & 120 & 31 & 25,83 \\ 55.250 & 16.334 & 29,56 & \text { España } & 3.740 & 999 & 26,71\end{array}$


«Papers»: Revista de Sociología

\begin{tabular}{lcccc}
\hline \multicolumn{2}{c}{ Alumnos matriculados } & \multicolumn{2}{c}{ Licenciados } \\
\hline Total Mujeres & $\%$ & Total & Mujeres & $\%$ \\
\hline
\end{tabular}

Facultad de Ciencias de la Información

\begin{tabular}{rrrlrrr}
867 & 270 & 31,14 & Autónoma & - & - & - \\
6.177 & 1.686 & 27,29 & España & - & - & - \\
\multicolumn{7}{c}{ Facultad de Filosofía } \\
9.422 & 4.999 & 53,05 & Central & 623 & 380 & 60,99 \\
2.764 & 1.650 & 59,69 & Autónoma & 113 & 69 & 61,06 \\
58.690 & 33.956 & 57,85 & España & 4.975 & 2.823 & 56,74
\end{tabular}

Escuela de $E G B$

$2.949 \quad 2.057 \quad 69,75$

Escuela de Arquitectura

$\begin{array}{rrrlrrr}3.134 & 366 & 11,67 & \text { Politécnico } & 147 & 13 & 8,84 \\ 45.768 & 1.923 & 4,20 & \text { España } & 556 & 32 & 5,75\end{array}$

Escuela de Ingenieros

$\begin{array}{rrrlrll}3.913 & 57 & 1,45 & \text { Politécnico } & 812 & 2 & 0,24 \\ 941 & 9 & 0,95 & \text { Tarrasa } & 58 & 0 & 0 \\ 13.631 & 164 & 1,20 & \text { España } & 1.639 & 8 & 0,48\end{array}$

Caminos, Canales y Puertos

386

4.892

$\begin{array}{rrll}5 & 1,29 & \text { Politécnico } & - \\ 44 & 0,89 & \text { España } & -\end{array}$

Telecomunicaciones

$\begin{array}{rrrllll}938 & 7 & 0,74 & \text { Politécnico } & - & - & - \\ 4.947 & 96 & 1,94 & \text { España } & - & - & -\end{array}$ 